J. Golak · R. Skibiński • H. Witała •

K. Topolnicki · W. Glöckle • A. Nogga •

H. Kamada

\title{
Different Methods for the Two- Nucleon T-Matrix in the Operator Form
}

the date of receipt and acceptance should be inserted later

\begin{abstract}
We compare three methods to calculate the nucleon-nucleon tmatrix based on the three-dimensional formulation of Ref. [1. In the first place we solve a system of complex linear inhomogeneous equations directly for the t-matrix. Our second method is based on iterations and a variant of the Lanczos algorithm. In the third case we obtain the t-matrix in two steps, solving a system of real linear equations for the k-matrix expansion coefficients and then solving an on-shell equation, which connects the scalar coefficients of the $\mathrm{k}$ - and t-matrices. A very good agreement among the three methods is demonstrated for selected nucleon-nucleon scattering observables using a chiral next-to-next-to-leading-order neutron-proton potential. We also apply our three-dimensional framework to the demanding problem of proton-proton scattering, using a corresponding version of the nucleon-nucleon potential and supplementing it with the (screened) Coulomb force, taken also in the three-dimensional form. We show converged results for two different screening functions and find a very good agreement with other methods dealing with proton-proton scattering.
\end{abstract}

J. Golak · R. Skibiński · H. Witała · K. Topolnicki

M. Smoluchowski Institute of Physics, Jagiellonian University, PL-30059 Kraków, Poland

W. Glöckle

Institut für Theoretische Physik II, Ruhr-Universität Bochum, D-44780 Bochum, Germany

A. Nogga

Forschungszentrum Jülich, Institut für Kernphysik, Institute for Advanced Simulation and Jülich Center for Hadron Physics, D-52425 Jülich, Germany

H. Kamada

Department of Physics, Faculty of Engineering, Kyushu Institute of Technology, 1-1 Sensuicho Tobata, Kitakyushu 804-8550, Japan 


\section{Introduction}

The nucleon-nucleon (NN) t-matrix appears not only in the description of the NN scattering process but constitutes a key ingredient of the three- and fournucleon calculations (see for example 2, 3, ). No wonder that many methods to calculate this quantity have been proposed and realized both in coordinate and in momentum space. For many years it has been natural to use the rotational invariance of the $\mathrm{NN}$ force and introduce a partial wave basis. This procedure has a clear physical meaning and is very accurate at low energies. However, at higher energies very many partial waves are necessary to achieve converged results, so methods employing vector variables might become more efficient.

There have been several approaches to NN scattering avoiding partial wave decomposition (PWD). Pioneering calculations of Ref. [4] carried out for different Malfliet-Tjon-type potentials gave interesting numerical insight in the properties of the three-dimensional (3D) t-matrix and paved the way for further investigations including spin degrees of freedom. A helicity formalism directly linked to the total NN spin was proposed in 5. Later, the spectator equation for relativistic NN scattering was solved in [6] also using a helicity formulation. Closely related are 3D formulations for pion-nucleon scattering [7] and for scattering of protons on light nuclei [8].

These developments were then used in few-body calculations. The Faddeev equation for the system of three bosons interacting via scalar forces was solved for the bound state case 9 as well as for three-body scattering [10 directly in three dimensions. The helicity formalism of Ref. 5 was employed in $3 \mathrm{~N}$ calculations in 11,12,13. Recently we proposed a 3D framework for $3 \mathrm{~N}$ bound 14, 15] and $3 \mathrm{~N}$ scattering states [16, where spin-momentum operators are introduced and treated analytically so the Faddeev equations turn into a finite set of coupled equations for scalar functions depending only on momentum vectors. For this formulation it is crucial that the most general form of the NN interaction can only depend on six linearly independent spinmomentum operators. In 1 we formulated a new approach to NN scattering and provided its numerical realization based on the matrix inversion method. A comparison with the standard PWD calculations for two quite different NN potentials and a few projectile energies proved that the new scheme is very accurate 1 .

However, in view of expected applications to the $3 \mathrm{~N}$ calculations, where we need the full off-shell t-matrix, we decided to develop a more efficient, iteration approach to calculate the 3D t-matrix. We also realized an old idea presented, for example, in [17] and prepared a scheme for obtaining the onshell t-matrix from the solution of the k-matrix equation.

In Sect. 2 we repeat briefly the main points of our approach starting from the most general form of the NN potential. Next we derive the corresponding equation for the k-matrix and formulate the subsequent equation for the on-shell t-matrix. Finally, we formulate our iteration scheme. Numerical realizations of our different approaches that employ a recent chiral next-to-nextleading order (NNLO) NN force [1, 19,20 are presented in Sect. 3. Here, in addition, we apply one of our schemes to the problem of proton-proton 
scattering (including the strong and screened Coulomb forces), and compare results for two different screening functions. We conclude in Sect. 4.

\section{The Formal Structure}

For the convenience of the reader we repeat here the main points of our formalism, introduced in Ref. [1. The NN potential in the two-nucleon $(2 \mathrm{~N})$ isospin space, spanned by the four states $\left|t m_{t}\right\rangle(|00\rangle,|1-1\rangle,|10\rangle,|11\rangle)$, is given as

$$
\left\langle t^{\prime} m_{t^{\prime}}|V| t m_{t}\right\rangle=\delta_{t t^{\prime}} \delta_{m_{t} m_{t^{\prime}}} V^{t m_{t}},
$$

allowing for charge independence and charge symmetry breaking. The most general rotational, parity and time reversal invariant isospin projected NN force is then expanded into six scalar spin-momentum operators $w_{i}\left(\boldsymbol{\sigma}_{1}, \boldsymbol{\sigma}_{2}, \mathbf{p}^{\prime}, \mathbf{p}\right)$ 21] as

$$
V^{t m_{t}}=\sum_{j=1}^{6} v_{j}^{t m_{t}}\left(\mathbf{p}^{\prime}, \mathbf{p}\right) w_{j}\left(\boldsymbol{\sigma}_{1}, \boldsymbol{\sigma}_{2}, \mathbf{p}^{\prime}, \mathbf{p}\right),
$$

using [1]

$$
\begin{aligned}
& w_{1}\left(\boldsymbol{\sigma}_{1}, \boldsymbol{\sigma}_{2}, \mathbf{p}^{\prime}, \mathbf{p}\right)=1, \\
& w_{2}\left(\boldsymbol{\sigma}_{1}, \boldsymbol{\sigma}_{2}, \mathbf{p}^{\prime}, \mathbf{p}\right)=\boldsymbol{\sigma}_{1} \cdot \boldsymbol{\sigma}_{2}, \\
& w_{3}\left(\boldsymbol{\sigma}_{1}, \boldsymbol{\sigma}_{2}, \mathbf{p}^{\prime}, \mathbf{p}\right)=i\left(\boldsymbol{\sigma}_{1}+\boldsymbol{\sigma}_{2}\right) \cdot\left(\mathbf{p} \times \mathbf{p}^{\prime}\right), \\
& w_{4}\left(\boldsymbol{\sigma}_{1}, \boldsymbol{\sigma}_{2}, \mathbf{p}^{\prime}, \mathbf{p}\right)=\boldsymbol{\sigma}_{1} \cdot\left(\mathbf{p} \times \mathbf{p}^{\prime}\right) \boldsymbol{\sigma}_{2} \cdot\left(\mathbf{p} \times \mathbf{p}^{\prime}\right), \\
& w_{5}\left(\boldsymbol{\sigma}_{1}, \boldsymbol{\sigma}_{2}, \mathbf{p}^{\prime}, \mathbf{p}\right)=\boldsymbol{\sigma}_{1} \cdot\left(\mathbf{p}^{\prime}+\mathbf{p}\right) \boldsymbol{\sigma}_{2} \cdot\left(\mathbf{p}^{\prime}+\mathbf{p}\right), \\
& w_{6}\left(\boldsymbol{\sigma}_{1}, \boldsymbol{\sigma}_{2}, \mathbf{p}^{\prime}, \mathbf{p}\right)=\boldsymbol{\sigma}_{1} \cdot\left(\mathbf{p}^{\prime}-\mathbf{p}\right) \boldsymbol{\sigma}_{2} \cdot\left(\mathbf{p}^{\prime}-\mathbf{p}\right),
\end{aligned}
$$

and scalar functions $v_{j}^{t m_{t}}\left(\mathbf{p}^{\prime}, \mathbf{p}\right)$ which depend only on the $\mathbf{p}$ and $\mathbf{p}^{\prime}$ momenta. A corresponding expansion into the $w_{i}\left(\boldsymbol{\sigma}_{1}, \boldsymbol{\sigma}_{2}, \mathbf{p}^{\prime}, \mathbf{p}\right)$ operators can be applied to the NN t-operator at the $2 \mathrm{~N}$ internal energy $z$ :

$$
t^{t m_{t}}(z)=\sum_{j=1}^{6} t_{j}^{t m_{t}}\left(\mathbf{p}^{\prime}, \mathbf{p} ; z\right) w_{j}\left(\boldsymbol{\sigma}_{1}, \boldsymbol{\sigma}_{2}, \mathbf{p}^{\prime}, \mathbf{p}\right) .
$$

The $z$ parameter appears in the Lippmann-Schwinger (LS) equation

$$
t^{t m_{t}}(z)=V^{t m_{t}}+V^{t m_{t}} G_{0}(z) t^{t m_{t}}(z),
$$

with $G_{0}(z)=\left(z-H_{0}\right)^{-1}$ being the free resolvent.

Inserting Eqs. (2) and (4) into the LS equation (5), operating with $w_{k}\left(\boldsymbol{\sigma}_{1}, \boldsymbol{\sigma}_{2}, \mathbf{p}^{\prime}, \mathbf{p}\right)$ from the left and performing the trace in the $\mathrm{NN}$ spin space leads to

$$
\begin{array}{r}
\sum_{j} A_{k j}\left(\mathbf{p}^{\prime}, \mathbf{p}\right) t_{j}^{t m_{t}}\left(\mathbf{p}^{\prime}, \mathbf{p} ; z\right)=\sum_{j} A_{k j}\left(\mathbf{p}^{\prime}, \mathbf{p}\right) v_{j}^{t m_{t}}\left(\mathbf{p}^{\prime}, \mathbf{p}\right) \\
+\int d^{3} p^{\prime \prime} \sum_{j j^{\prime}} v_{j}^{t m_{t}}\left(\mathbf{p}^{\prime}, \mathbf{p}^{\prime \prime}\right) \frac{1}{z-\frac{p^{\prime \prime 2}}{m}+i \epsilon} t_{j^{\prime}}^{t m_{t}}\left(\mathbf{p}^{\prime \prime}, \mathbf{p} ; z\right) B_{k j j^{\prime}}\left(\mathbf{p}^{\prime}, \mathbf{p}^{\prime \prime}, \mathbf{p}\right) .
\end{array}
$$


The scalar coefficients $A_{k j}$ and $B_{k j j^{\prime}}$, defined as

$$
\begin{aligned}
A_{k j}\left(\mathbf{p}^{\prime}, \mathbf{p}\right) \equiv & \operatorname{Tr}\left(w_{k}\left(\boldsymbol{\sigma}_{1}, \boldsymbol{\sigma}_{2}, \mathbf{p}^{\prime}, \mathbf{p}\right) w_{j}\left(\boldsymbol{\sigma}_{1}, \boldsymbol{\sigma}_{2}, \mathbf{p}^{\prime}, \mathbf{p}\right)\right), \\
B_{k j j^{\prime}}\left(\mathbf{p}^{\prime}, \mathbf{p}^{\prime \prime}, \mathbf{p}\right) \equiv & \operatorname{Tr}\left(w_{k}\left(\boldsymbol{\sigma}_{1}, \boldsymbol{\sigma}_{2}, \mathbf{p}^{\prime}, \mathbf{p}\right) w_{j}\left(\boldsymbol{\sigma}_{1}, \boldsymbol{\sigma}_{2}, \mathbf{p}^{\prime}, \mathbf{p}^{\prime \prime}\right)\right. \\
& \left.\times w_{j^{\prime}}\left(\boldsymbol{\sigma}_{1}, \boldsymbol{\sigma}_{2}, \mathbf{p}^{\prime \prime}, \mathbf{p}\right)\right),
\end{aligned}
$$

are given explicitly in Appendix A of Ref. [1. In this way we obtain a set of six coupled equations for the scalar functions $t_{j}^{t m_{t}}\left(\mathbf{p}^{\prime}, \mathbf{p} ; z\right)$, which depend, for fixed $z$ and $|\mathbf{p}|$, on two variables, $\left|\mathbf{p}^{\prime}\right|$ and the cosine of the relative angle between the vectors $\mathbf{p}^{\prime}$ and $\mathbf{p}$, given by $\hat{\mathbf{p}}^{\prime} \cdot \hat{\mathbf{p}}$.

In Ref. 1] we showed all steps leading to the NN elastic scattering observables. The LS equation was solved there for positive energy of the NN system, $z \equiv E_{c . m} . \equiv \frac{p_{0}^{2}}{m}$, where $m$ is the nucleon mass. We chose the following representation for the vectors $\hat{\mathbf{p}}, \hat{\mathbf{p}}^{\prime}$ and $\hat{\mathbf{p}}^{\prime \prime}$ :

$$
\begin{aligned}
\hat{\mathbf{p}} & =(0,0,1), \\
\hat{\mathbf{p}}^{\prime} & =\left(\sqrt{1-x^{\prime 2}}, 0, x^{\prime}\right), \\
\hat{\mathbf{p}}^{\prime \prime} & =\left(\sqrt{1-x^{\prime \prime}} \cos \varphi^{\prime \prime}, \sqrt{1-x^{\prime \prime 2}} \sin \varphi^{\prime \prime}, x^{\prime \prime}\right),
\end{aligned}
$$

and prepared grids for the different variables in the problem. We took $n_{x}$ Gaussian points for the $x^{\prime \prime}$ integration and used the same grid for the $x^{\prime}$ points. We employed $n_{p}$ Gaussian points for the $p^{\prime}$ and $p^{\prime \prime}$ grids, which are defined in the interval $(0, \bar{p})$. In addition, $p_{0}$ is added to the set of $p^{\prime}$ points. The intermediate $\varphi^{\prime \prime}$ integration was performed with $n_{\varphi^{\prime \prime}}$ Gaussian points. Thus, from Eq. (6) we got a system of $6 \times\left(n_{p}+1\right) \times n_{x}$ linear equations for given $p_{0}$ and fixed $p$. In Ref. [1] we set from the very beginning $p=p_{0}$, so the solution contained the on-shell t-matrix in the operator form, $t_{j}\left(p_{0}, p_{0}, x^{\prime} ; E_{c . m .}\right)$. Since the six operators (3) are on-shell linearly dependent, our solutions were not unique. They always led, however, to stable and unique predictions for the observables. In Ref. 1 we worked with the standard LU decomposition of Numerical Recipes 22] for two different NN forces (the Bonn B [23] potential and a chiral NNLO potential [18,19,20]) and made calculations for several $E_{c . m}$. energies. For all considered cases we obtained a perfect agreement between this new 3D approach and the calculations based on standard partial wave methods.

Solving Eq. (6) directly as a system of inhomogeneous coupled algebraic equations is not efficient. Especially in view of the applications in the threenucleon system, where a full off-shell t-matrix is needed, it is worth looking for a faster method. A typical job employing $n_{x}=36, n_{p}=36$ and $n_{\varphi^{\prime \prime}}=60$ Gaussian points might take a few hours on a PC. In Ref. [1] we tried to solve Eq. (6) by iterations. In the matrix form this equation was written as

$$
A t=A v+B t
$$


where $t_{j}\left(p^{\prime}, p, x^{\prime} ; z\right)\left(v_{j}\left(p^{\prime}, p, x^{\prime}\right)\right)$ constituted the components of the $t(v)$ vector and

$$
(B t)_{k}\left(p^{\prime}, p, x^{\prime}\right) \equiv \int_{0}^{\bar{p}} d p^{\prime \prime} p^{\prime \prime 2} \frac{1}{p_{0}^{2}-p^{\prime \prime 2}+i \epsilon} f_{k}\left(p^{\prime \prime} ; p^{\prime}, p, x^{\prime}\right),
$$

with

$f_{k}\left(p^{\prime \prime} ; p^{\prime}, p, x^{\prime}\right) \equiv$

$m \sum_{j, j^{\prime}=1}^{6} \int_{-1}^{1} d x^{\prime \prime} \int_{0}^{2 \pi} d \varphi^{\prime \prime} B_{k j j^{\prime}}\left(p^{\prime}, p^{\prime \prime}, p, x^{\prime}, x^{\prime \prime}, \varphi^{\prime \prime}\right) v_{j}\left(p^{\prime}, p^{\prime \prime}, y\right) t_{j^{\prime}}\left(p^{\prime \prime}, p, x^{\prime \prime}\right)$,

where

$$
y \equiv x^{\prime} x^{\prime \prime}+\sqrt{1-x^{\prime 2}} \sqrt{1-x^{\prime \prime 2}} \cos \varphi^{\prime \prime} .
$$

It was possible to use such $p, p^{\prime}$ and $x^{\prime}$ points that the matrix $A$ could be inverted [1]. In this case Eq. (6) was written as

$$
t\left(p^{\prime}, p, x^{\prime}\right)=v\left(p^{\prime}, p, x^{\prime}\right)+A^{-1}\left(p^{\prime}, p, x^{\prime}\right)(B t)\left(p^{\prime}, p, x^{\prime}\right)
$$

so we arrived at the following iteration scheme:

$$
\begin{aligned}
t^{(1)}\left(p^{\prime}, p, x^{\prime}\right) & =v\left(p^{\prime}, p, x^{\prime}\right), \\
t^{(n)}\left(p^{\prime}, p, x^{\prime}\right) & =v\left(p^{\prime}, p, x^{\prime}\right) \\
& +A^{-1}\left(p^{\prime}, p, x^{\prime}\right)\left(B t^{(n-1)}\right)\left(p^{\prime}, p, x^{\prime}\right), \text { for } n>1 .
\end{aligned}
$$

We found it, however, very difficult to maintain numerical stability for this iterative method. Fortunately, it turned out that our scheme could be easily modified to give stable results. It is sufficient to combine, on the level of analytical calculations, the $A_{k j}^{-1}$ and $B_{k j j^{\prime}}$ coefficients, preparing

$$
\tilde{B}_{k j j^{\prime}}\left(\mathbf{p}^{\prime}, \mathbf{p}^{\prime \prime}, \mathbf{p}\right) \equiv \sum_{l=1}^{6} A_{k l}^{-1}\left(\mathbf{p}^{\prime}, \mathbf{p}\right) B_{l j j^{\prime}}\left(\mathbf{p}^{\prime}, \mathbf{p}^{\prime \prime}, \mathbf{p}\right) .
$$

The new, numerically safe, iteration scheme reads thus:

$$
\begin{aligned}
& t^{(1)}\left(p^{\prime}, p, x^{\prime}\right)=v\left(p^{\prime}, p, x^{\prime}\right) \\
& t^{(n)}\left(p^{\prime}, p, x^{\prime}\right)=v\left(p^{\prime}, p, x^{\prime}\right)+\left(\tilde{B} t^{(n-1)}\right)\left(p^{\prime}, p, x^{\prime}\right), \text { for } n>1,
\end{aligned}
$$

where

$$
(\tilde{B} t)_{k}\left(p^{\prime}, p, x^{\prime}\right) \equiv \int_{0}^{\bar{p}} d p^{\prime \prime} p^{\prime \prime 2} \frac{1}{p_{0}^{2}-p^{\prime \prime 2}+i \epsilon} \tilde{f}_{k}\left(p^{\prime \prime} ; p^{\prime}, p, x^{\prime}\right)
$$


and

$\tilde{f}_{k}\left(p^{\prime \prime} ; p^{\prime}, p, x^{\prime}\right) \equiv$

$m \sum_{j, j^{\prime}=1}^{6} \int_{-1}^{1} d x^{\prime \prime} \int_{0}^{2 \pi} d \varphi^{\prime \prime} \tilde{B}_{k j j^{\prime}}\left(p^{\prime}, p^{\prime \prime}, p, x^{\prime}, x^{\prime \prime}, \varphi^{\prime \prime}\right) v_{j}\left(p^{\prime}, p^{\prime \prime}, y\right) t_{j^{\prime}}\left(p^{\prime \prime}, p, x^{\prime \prime}\right)$.

The above given iterative method proves also very fast. It is very important from the numerical point of view that the summation over $j$ and the integral over $\varphi^{\prime \prime}$ do not involve $t_{j}\left(p^{\prime}, p, x^{\prime}\right)$, which means that they can be prepared and stored in advance and later used in each iteration. Furthermore, this scheme can be easily implemented on a parallel machine and used to calculate $t_{j}\left(p^{\prime}, p, x^{\prime}\right)$ for many $p$ points at one shot. In the case, when one is interested in the on-shell t-matrix, it is impossible to set directly $p=p_{0}$, if the $p^{\prime}$ points contain $p_{0}$. It is sufficient to make calculations for two $p$ values, $p=p_{0} \pm \delta_{p_{0}}$ $\left(\delta_{p_{0}} \approx 0.01 \mathrm{fm}^{-1}\right)$ and then take the average:

$$
\begin{aligned}
t_{j}\left(p_{0}, p_{0}, x^{\prime} ; z=\frac{p_{0}^{2}}{m}\right) \approx & \frac{1}{2}\left(t_{j}\left(p_{0}, p_{0}-\delta_{p_{0}}, x^{\prime} ; z=\frac{p_{0}^{2}}{m}\right)\right. \\
& \left.+t_{j}\left(p_{0}, p_{0}+\delta_{p_{0}}, x^{\prime} ; z=\frac{p_{0}^{2}}{m}\right)\right) .
\end{aligned}
$$

This method was successfully employed in the recent $3 \mathrm{~N}$ bound state calculations 15. In this case the kernel contains no pole, which makes the calculations even faster. We use a variant of the Lanczos method [24] to sum the resulting Neumann series, which proves to be more accurate than the standard Padé scheme.

Alternatively, in many calculations using PWD of the NN potential, the on-shell t-matrix is obtained not directly but in two steps. First one defines the k-matrix through the principal value kernel [17]

$$
\begin{aligned}
& \left\langle\mathbf{p}^{\prime}\left|k^{t m_{t}}(z)\right| \mathbf{p}\right\rangle=\left\langle\mathbf{p}^{\prime}\left|V^{t m_{t}}\right| \mathbf{p}\right\rangle \\
& +\int d^{3} p^{\prime \prime}\left\langle\mathbf{p}^{\prime}\left|V^{t m_{t}}\right| \mathbf{p}^{\prime \prime}\right\rangle \frac{P}{z-\frac{p^{\prime \prime 2}}{m}}\left\langle\mathbf{p}^{\prime \prime}\left|k^{t m_{t}}(z)\right| \mathbf{p}\right\rangle
\end{aligned}
$$

and the connection to the t-matrix is given as

$$
\begin{aligned}
& \left\langle\mathbf{p}^{\prime}\left|t^{t m_{t}}(z)\right| \mathbf{p}\right\rangle=\left\langle\mathbf{p}^{\prime}\left|k^{t m_{t}}(z)\right| \mathbf{p}\right\rangle \\
& -i \pi \int d^{3} p^{\prime \prime}\left\langle\mathbf{p}^{\prime}\left|k^{t m_{t}}(z)\right| \mathbf{p}^{\prime \prime}\right\rangle \delta\left(z-\frac{p^{\prime \prime 2}}{m}\right)\left\langle\mathbf{p}^{\prime \prime}\left|t^{t m_{t}}(z)\right| \mathbf{p}\right\rangle .
\end{aligned}
$$

If we take $z=\frac{p_{0}^{2}}{m},\left|\mathbf{p}^{\prime \prime}\right|=\left|\mathbf{p}^{\prime}\right|=|\mathbf{p}|=p_{0}$, then we get the following (on shell) relation

$$
\begin{aligned}
& \left\langle\mathbf{p}^{\prime}\left|t^{t m_{t}}(z)\right| \mathbf{p}\right\rangle=\left\langle\mathbf{p}^{\prime}\left|k^{t m_{t}}(z)\right| \mathbf{p}\right\rangle \\
& -\frac{i}{2} \pi m p_{0} \int d \hat{p}^{\prime \prime}\left\langle\mathbf{p}^{\prime}\left|k^{t m_{t}}(z)\right| \mathbf{p}^{\prime \prime}\right\rangle\left\langle\mathbf{p}^{\prime \prime}\left|t^{t m_{t}}(z)\right| \mathbf{p}\right\rangle,
\end{aligned}
$$


where $d \hat{p}^{\prime \prime}$ denotes the two-dimensional angular integration.

Including spin degrees of freedom and using the expansion of the k-matrix into the $w_{i}\left(\boldsymbol{\sigma}_{1}, \boldsymbol{\sigma}_{2}, \mathbf{p}^{\prime}, \mathbf{p}\right)$ operators (3), it is easy to find the equation corresponding to (21) but for the scalar coefficients of the k-matrix, $k_{j}^{t m_{t}}\left(\mathbf{p}^{\prime}, \mathbf{p} ; z\right)$ :

$$
\begin{array}{r}
\sum_{j} A_{k j}\left(\mathbf{p}^{\prime}, \mathbf{p}\right) k_{j}^{t m_{t}}\left(\mathbf{p}^{\prime}, \mathbf{p} ; z\right)=\sum_{j} A_{k j}\left(\mathbf{p}^{\prime}, \mathbf{p}\right) v_{j}^{t m_{t}}\left(\mathbf{p}^{\prime}, \mathbf{p}\right) \\
+\int d^{3} p^{\prime \prime} \sum_{j j^{\prime}} v_{j}^{t m_{t}}\left(\mathbf{p}^{\prime}, \mathbf{p}^{\prime \prime}\right) \frac{P}{z-\frac{p^{\prime \prime 2}}{m}} k_{j^{\prime}}^{t m_{t}}\left(\mathbf{p}^{\prime \prime}, \mathbf{p} ; z\right) B_{k j j^{\prime}}\left(\mathbf{p}^{\prime}, \mathbf{p}^{\prime \prime}, \mathbf{p}\right) .
\end{array}
$$

The coefficients $A_{k j}$ and $B_{k j j^{\prime}}$ are the same as in Eq. (6). By simple interpolation or by taking the average over two $p$ values, we get the on-shell k-matrix coefficients, $k_{j}^{t m_{t}}\left(p_{0}, p_{0}, x^{\prime} ; \frac{p_{0}^{2}}{m}\right)$. In order to formulate an equation corresponding to (23), we have to take into account that on-shell there are only five independent $w_{i}\left(\boldsymbol{\sigma}_{1}, \boldsymbol{\sigma}_{2}, \mathbf{p}^{\prime}, \mathbf{p}\right)$ operators. This follows directly from the (on shell) relation

$$
\begin{aligned}
\boldsymbol{\sigma}_{1} \cdot \boldsymbol{\sigma}_{2} & =\frac{1}{\left(\mathbf{p} \times \mathbf{p}^{\prime}\right)^{2}} \boldsymbol{\sigma}_{1} \cdot\left(\mathbf{p} \times \mathbf{p}^{\prime}\right) \boldsymbol{\sigma}_{2} \cdot\left(\mathbf{p} \times \mathbf{p}^{\prime}\right) \\
& +\frac{1}{\left(\mathbf{p}+\mathbf{p}^{\prime}\right)^{2}} \sigma_{1} \cdot\left(\mathbf{p}+\mathbf{p}^{\prime}\right) \boldsymbol{\sigma}_{2} \cdot\left(\mathbf{p}+\mathbf{p}^{\prime}\right) \\
& +\frac{1}{\left(\mathbf{p}-\mathbf{p}^{\prime}\right)^{2}} \boldsymbol{\sigma}_{1} \cdot\left(\mathbf{p}-\mathbf{p}^{\prime}\right) \boldsymbol{\sigma}_{2} \cdot\left(\mathbf{p}-\mathbf{p}^{\prime}\right),
\end{aligned}
$$

and can be written as

$$
w_{2}=\frac{1}{p_{0}^{4}\left(1-x^{\prime 2}\right)} w_{4}+\frac{1}{2 p_{0}^{2}\left(1+x^{\prime}\right)} w_{5}+\frac{1}{2 p_{0}^{2}\left(1-x^{\prime}\right)} w_{6} .
$$

Using Eq. (26) it is easy to write the on-shell k-matrix as a linear combination of only five operators. Then the on shell equation corresponding to (23) takes the form

$$
\begin{aligned}
& \sum_{j=1}^{5} A_{k j}\left(p_{0}, p_{0}, x^{\prime}\right) t_{j}^{t m_{t}}\left(p_{0}, p_{0}, x^{\prime} ; \frac{p_{0}^{2}}{m}\right)= \\
& \sum_{j=1}^{5} A_{k j}\left(p_{0}, p_{0}, x^{\prime}\right) k_{j}^{t m_{t}}\left(p_{0}, p_{0}, x^{\prime} ; \frac{p_{0}^{2}}{m}\right) \\
& -\frac{i}{2} \pi m p_{0} \int_{-1}^{1} d x^{\prime \prime} \int_{0}^{2 \pi} d \varphi^{\prime \prime} \sum_{j, j^{\prime}=1}^{5} k_{j}^{t m_{t}}\left(p_{0}, p_{0}, y ; \frac{p_{0}^{2}}{m}\right) \\
& \times t_{j^{\prime}}^{t m_{t}}\left(p_{0}, p_{0}, x^{\prime \prime} ; \frac{p_{0}^{2}}{m}\right) B_{k j j^{\prime}}\left(p_{0}, p_{0}, p_{0}, x^{\prime}, x^{\prime \prime}, \varphi^{\prime \prime}\right) .
\end{aligned}
$$

In principle, any operator $w_{i}$ appearing in (26) can be eliminated but not all the possible choices are numerically equivalent due to different behavior of the resulting coefficients in the vicinity of $x^{\prime}= \pm 1$. This is demonstrated in Sect. 3. In the case when the operator $w_{4}$ or $w_{5}$ is removed, the $A_{k j}$ and $B_{k j j^{\prime}}$ coefficients in Eq. (27) have to be renumbered accordingly. 


\section{Numerical Results}

In this section we focus on the on-shell t-matrix obtained without employing PWD. We do not compare our 3D results with the results obtained by standard momentum space PWD, since this comparison was carried out successfully in [1].

We show first that the methods discussed in the previous section lead to stable numerical results. We use the matrix inversion scheme, introduced already in 1. In present calculations we use a parallel version of the matrix inversion, based on the SCALAPACK library [26]. We implement also the iterative algorithm and sum up the resulting Neumann series using a variant of the Lanczos method 24. Finally, we solve the equation for the k-matrix and, going on-shell, eliminate either the $w_{6}$ or the $w_{4} 2 \mathrm{~N}$ operator.

We perform calculations using all these methods for two projectile laboratory kinetic energies, 13 and $300 \mathrm{MeV}$. We use first the neutron-proton version of a chiral NNLO NN potential [18, 19,20, described briefly in Appendix A of Ref. [1].

In Figs. 1 and 2 we show results for several NN scattering observables. In the case of $13 \mathrm{MeV}$, the agreement among the four types of results is very good both for the neutron-neutron (left panel) and the neutron-proton (right panel) system. For the energy of $300 \mathrm{MeV}$ we see that the calculations using the k-matrix equation without the $w_{6}$ operator on-shell, yield slightly different results for the $R$ and $A$ observables in the case of the neutron-proton system, than the other three schemes. These numerical instabilities become better visible for the Wolfenstein parameters $17 \mathrm{c}, g, h$ and $m$ shown in Fig. 3. Thus this particular numerical scheme should be avoided.

In the case, where iterations are used, we wanted to know how many iterations are needed to achieve full convergence of our results. We expected that the convergence depends on the NN system internal energy and show in Figs 4 and 5 our results for various numbers of iterations again for the small $(13 \mathrm{MeV})$ and large $(300 \mathrm{MeV})$ laboratory energy. At $13 \mathrm{MeV}$ and for the neutron-neutron system we find that 10 iterations are fully sufficient. For some observables results obtained with 8 iterations are very close to the predictions based on 20 iterations. For the neutron-proton case one needs generally 12 iterations. At $300 \mathrm{MeV}$ we see even faster convergence. For the neutron-neutron case predictions based on 6 iterations have already a correct shape (this was generally not the case for $13 \mathrm{MeV}$ ) and 8 iterations bring us very close to the fully converged results, although some tiny deviations are still visible. For the neutron-proton system results obtained from 10 iterations are still slightly different from the fully converged predictions. We can thus state that for energies below the pion production threshold, one needs no more than 12 iterations to arrive at fully converged results. Since the essential part of the iteration kernel can be prepared in advance, iterations run very fast, especially for the case of negative $2 \mathrm{~N}$ internal energy, where no singularity is present. This method is considered by us as the best for $3 \mathrm{~N}$ calculations.

In the remaining part of this section we consider the most difficult case in the nucleon-nucleon scattering, namely the proton-proton case with inclusion 
of the Coulomb force. As described in Ref. [27, contrary to the approaches using the so-called renormalization procedure (see for example [ 8 ]), we add a screened Coulomb potential to the (strong) proton-proton $2 \mathrm{~N}$ force given in the operator form and use this sum as input in our 3D calculations. We work with the proton-proton version of the same chiral NNLO $2 \mathrm{~N}$ potential and choose a relatively small laboratory energy $(13 \mathrm{MeV})$, where Coulomb effects are expected to play an important role. We assent to two types of screening, the exponential screening, $s_{1}(r ; n, R)$,

$$
s_{1}(r ; n, R) \equiv \exp \left(-\left(\frac{r}{R}\right)^{n}\right)
$$

restricting ourselves to the case $n=4$ and the localized (transition from 1 to 0 takes place in a finite interval) screening, $s_{2}(r ; R)$, proposed in 8$]$ :

$$
s_{2}(r ; R) \equiv \theta(R-r)+\frac{1}{2} \theta(r-R) \theta(3 R-r)\left(\sin \left(\frac{\pi r}{2 R}\right)+1\right)
$$

with $\theta(x)$ being the (unit) step function. The 3D momentum space screened Coulomb potential is obtained as in Ref. 28 .

In Figs. 6] and 7 we demonstrate a convergence of the results for some observables with the increasing parameter $R$. In the case of the exponential screening with $n=4$ it is achieved for $R=120 \mathrm{fm}$, while for the second type of screening $R=55 \mathrm{fm}$ is sufficient. In Fig. 8 we show that, as expected, the two screening prescriptions lead to the same final result for the selected proton-proton observables in our fully $3 \mathrm{D}$ calculations. The inset in Fig. 8 serves to compare the two screening functions for the parameters used in the actual calculations.

Proton-proton scattering was already considered by us in [27]. There we used the Vincent-Phatak method 29 and the method combining PWD calculations with the full 3D t-matrix of the screened Coulomb potential. Using those two methods, we performed calculations for the proton-proton strong potential and screening functions used in this paper. Predictions for the $R$ parameters yielding converged results in the case of the fully $3 \mathrm{D}$ calculations (solid line) are compared in Figs 9 (exponential screening) and 10 (localized screening) with the Vincent-Phatak method (dotted line) and the method combining PWD and 3D approaches (dashed line). We observe a good or even a very good agreement among all the methods for the polarization observables, especially for the exponential screening. Discrepancies in all the cases, including the most visible ones for the cross section, do not exceed 1$2 \%$. This agreement provides yet another proof that our numerical treatment of nucleon-nucleon scattering is fully reliable.

\section{Summary and conclusions}

In 11 we formulated an approach to the NN system employing spin-momentum operators multiplied by scalar functions, which only depend on the momentum vectors. This representation of the NN potential leads to a system of six coupled equations for the scalar functions defining the NN t-matrix. This system of equations was first solved using the matrix inversion method. 
In the present paper we formulate two other methods to calculate the nucleon-nucleon t-matrix in the same 3D formulation of Ref. 11. Our second method is iterative and uses a variant of the Lanczos algorithm 24 . In addition, as a third possibility, we treat the equation for the k-matrix and obtain the on-shell t-matrix coefficients, solving an additional equation, which connects the on-shell scalar coefficients of the $\mathrm{k}$ - and t-matrices. In the second step care is required, because only five spin-momentum operators are independent and not all choices are numerically safe. We show a very good agreement among all the considered methods for the selected neutronneutron and neutron-proton observables. In these calculations we use the neutron-proton version of a chiral NNLO potential. In the case of the iteration method we demonstrate also a fast convergence with respect to the number of iterations. This iterative method is best suited to be used in the $3 \mathrm{~N}$ bound state and in the $3 \mathrm{~N}$ continuum calculations. In fact it has been already successfully used in the $3 \mathrm{~N}$ bound state calculations [15, realizing the theoretical formulation given in [14].

Last not least, we apply our 3D framework to the problem of protonproton scattering, using the proton-proton version of the chiral NNLO NN potential, supplemented with the (screened) Coulomb force. We show converged results for two different screening functions and find a good agreement with two other methods considered in [27].

\section{Acknowledgments}

We thank Dr. Evgeny Epelbaum for providing us with a code for the operator form of the chiral neutron-proton and proton-proton NNLO potentials.

We acknowledge support by the Foundation for Polish Science - MPD program, co-financed by the European Union within the Regional Development Fund.

This work was supported by the Polish National Science Center under Grant No. DEC-2011/01/B/ST2/00578. It was also partially supported by the European Community-Research Infrastructure Integrating Activity "Exciting Physics Of Strong Interactions" (acronym WP4 EPOS) under the Seventh Framework Programme of EU. The numerical calculations were partly performed on the supercomputer cluster of the JSC, Jülich, Germany.

\section{References}

1. Golak,J., Glöckle, W., Skibiński,R., Witała,H., Rozpędzik,D., Topolnicki,K., Fachruddin,I., Elster,Ch., Nogga,A.: Two-nucleon systems in three dimensions. Phys. Rev. C81, 034006, (2010)

2. Glöckle,W., Witała,H., Hüber,D., Kamada,H., Golak,J.: The three-nucleon continuum: achievements, challenges and applications. Phys. Rep. 274, 107 (1996)

3. Nogga,A., Kamada,H., Glöckle,W.: Modern nuclear force predictions for the alpha particle. Phys. Rev. Lett. 85, 944 (2000)

4. Elster,Ch., Thomas,J.H., Glöckle,W.: Two-Body T-matrices Without AngularMomentum Decomposition:Energy and Momentum Dependences. Few-Body Syst. 24, 55 (1998) 
5. Fachruddin,I., Elster,Ch., Glöckle,W., New forms of deuteron equations and wave function representations Phys. Rev. C63, 054003 (2001)

6. Ramalho,G., Arriaga,A., Peña,M.T.: Solution Of The Spectator Equation For Relativistic NN Scattering Without Partial Wave Expansion. Few-Body Syst. 39, 123 (2006)

7. Caia,G., Pascalutsa,V., Wright,L.E.: Solving potential scattering equations without partial wave decomposition. Phys. Rev. C69, 034003 (2004)

8. Rodriguez-Gallardo,M., Deltuva,A., Cravo,E., Crespo,R., Fonseca,A.C.: Twobody scattering without angular-momentum decomposition. Phys. Rev. C78, 034602 (2008)

9. Elster,Ch., Schadow,W., Nogga,A., Glöckle,W.: Three Body Bound State Calculations without Angular Momentum Decomposition. Few-Body Syst. 27, 83 (1999)

10. Liu,H., Elster,Ch., Glöckle,W.: Three-Body Scattering at Intermediate Energies. Phys. Rev. C72, 054003 (2005)

11. Bayegan,S., Hadizadeh,M.R., Harzchi,M., Three-nucleon bound state in a spinisospin dependent three dimensional approach. Phys. Rev. C77, 064005 (2008)

12. Bayegan,S., Shalchi,M.A., Hadizadeh,M.R.: Three dimensional calculations of NN bound and scattering states with a chiral potential up to N3LO. Phys. Rev. C79, 057001 (2009)

13. Hadizadeh,M.R., Tomio,Lauro, Bayegan,S.: Solutions of the bound-state Faddeev-Yakubovsky equations in three dimensions by using NN and $3 \mathrm{~N}$ potential models. Phys. Rev. C83, 054004 (2011)

14. Glöckle,W., Elster,Ch., Golak,J., Skibiński,R., Witała,H., Kamada,H.: A New Treatment of $2 \mathrm{~N}$ and 3N Bound States in Three Dimensions. Few-Body Syst. 47, 25 (2010)

15. Golak,J., Topolnicki,K., Skibiński,R., Glöckle,W., Kamada,H., Nogga,A.: A Three-Dimensional Treatment of the Three-Nucleon Bound State. in preparation

16. Glöckle,W., Fachruddin,I., Elster,Ch., Golak,J., Skibiński,R., Witała,H.: 3N Scattering in a Three-Dimensional Operator Formulation. Eur. Phys. J. A43, (2010) 339

17. Glöckle,W.:The Quantum Mechanical Few-Body Problem. Springer, Berlin (1983)

18. Epelbaum,E., Glöckle,W., Meißner,Ulf-G.: The two-nucleon system at next-tonext-to-next-to-leading order. Nucl. Phys. A747, 362 (2005)

19. Epelbaum,E.: Few-Nucleon Forces and Systems in Chiral Effective Field Theory. Prog. Part. Nucl. Phys. 57, 654 (2006)

20. Epelbaum,E., Hammer,H.W., Meißner,Ulf-G.: Modern Theory of Nuclear Forces. Rev. Mod. Phys. 81, 1773 (2009)

21. Wolfenstein,L.: Possible triple scattering experiments. Phys. Rev. 96, 1654 (1954)

22. Press,W., Flannery,B., Teukolsky,S., Vetterling,W.:Numerical Recipes. Cambridge University Press (1989)

23. Machleidt,R. Adv. Nucl. Phys. 19, 189 (1989)

24. Stadler, A., Glöckle, W., Sauer, P.U.: Faddeev Equations With Three-Nucleon Force In Momentum Space, Phys. Rev. C44, 2319 (1991)

25. Epelbaum.E.: private communication

26. ScaLAPACK Home Page: http://www.netlib.org/scalapack/scalapack_home.html

27. Skibiński,R., Golak,J., Witała,H., Glockle,W.: Proton-proton scattering without Coulomb force renormalization. Eur. Phys. J. A40, 215 (2009)

28. Skibiński,R., Golak,J., Witała,H.: Numerical investigations of the threedimensional proton-proton screened Coulomb t-matrix. Acta Phys. Polon. B41, 875 (2010)

29. Vincent,C.M., Phatak,S.C.: Accurate momentum-space method for scattering by nuclear and Coulomb potentials. Phys. Rev. C10, 391 (1974) 

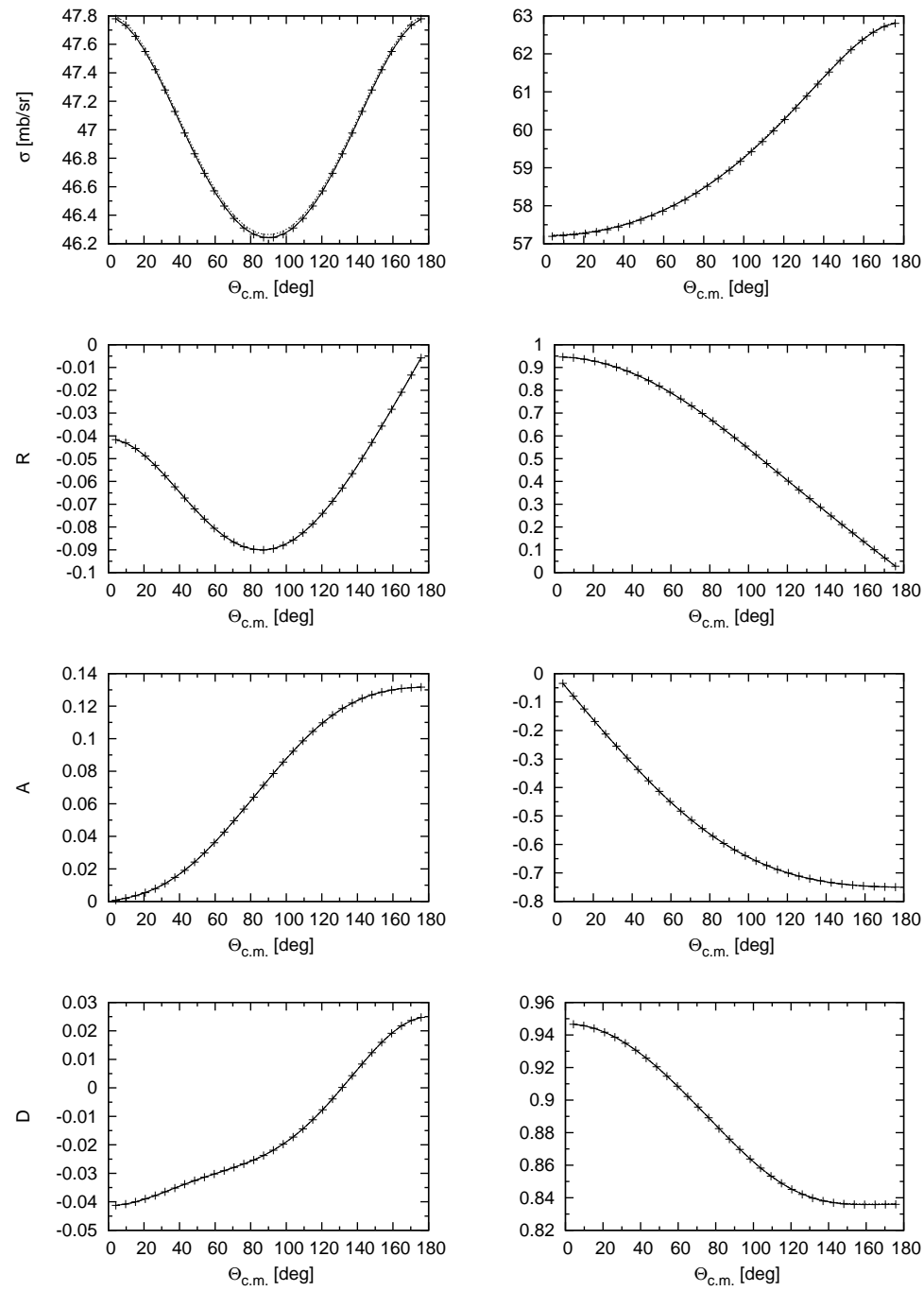

Fig. 1 Selected observables for the neutron-neutron (left panel) and neutronproton (right panel) system at the projectile laboratory kinetic energy $13 \mathrm{MeV}$ as a function of the center-of-mass angle $\theta_{c . m}$. for the chiral NNLO potential [19]. Crosses represent results obtained with the iterative method (see text). Dashed (dotted) lines depict the results obtained by solving first the equation for the $\mathrm{k}$ matrix and then the on-shell equation for the t-matrix, where on shell the $w_{4}\left(w_{6}\right)$ $2 \mathrm{~N}$ operator is eliminated. Solid lines are for the predictions based on the matrix inversion method. For the definition of the $R, A$ and $D$ observables see e.g. [17. 

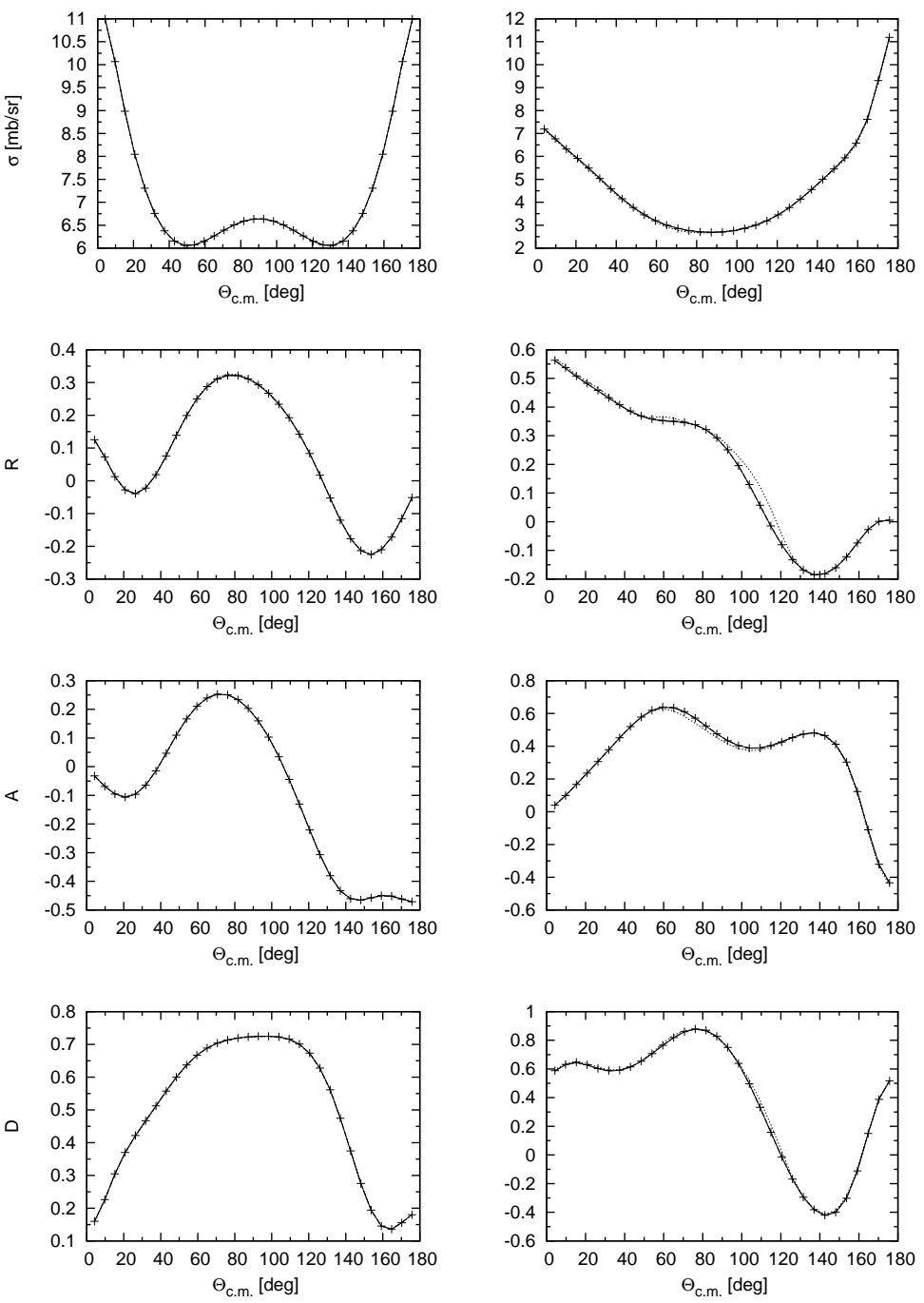

Fig. 2 The same as in Fig. 11 for the projectile laboratory kinetic energy $E=300 \mathrm{MeV}$ 

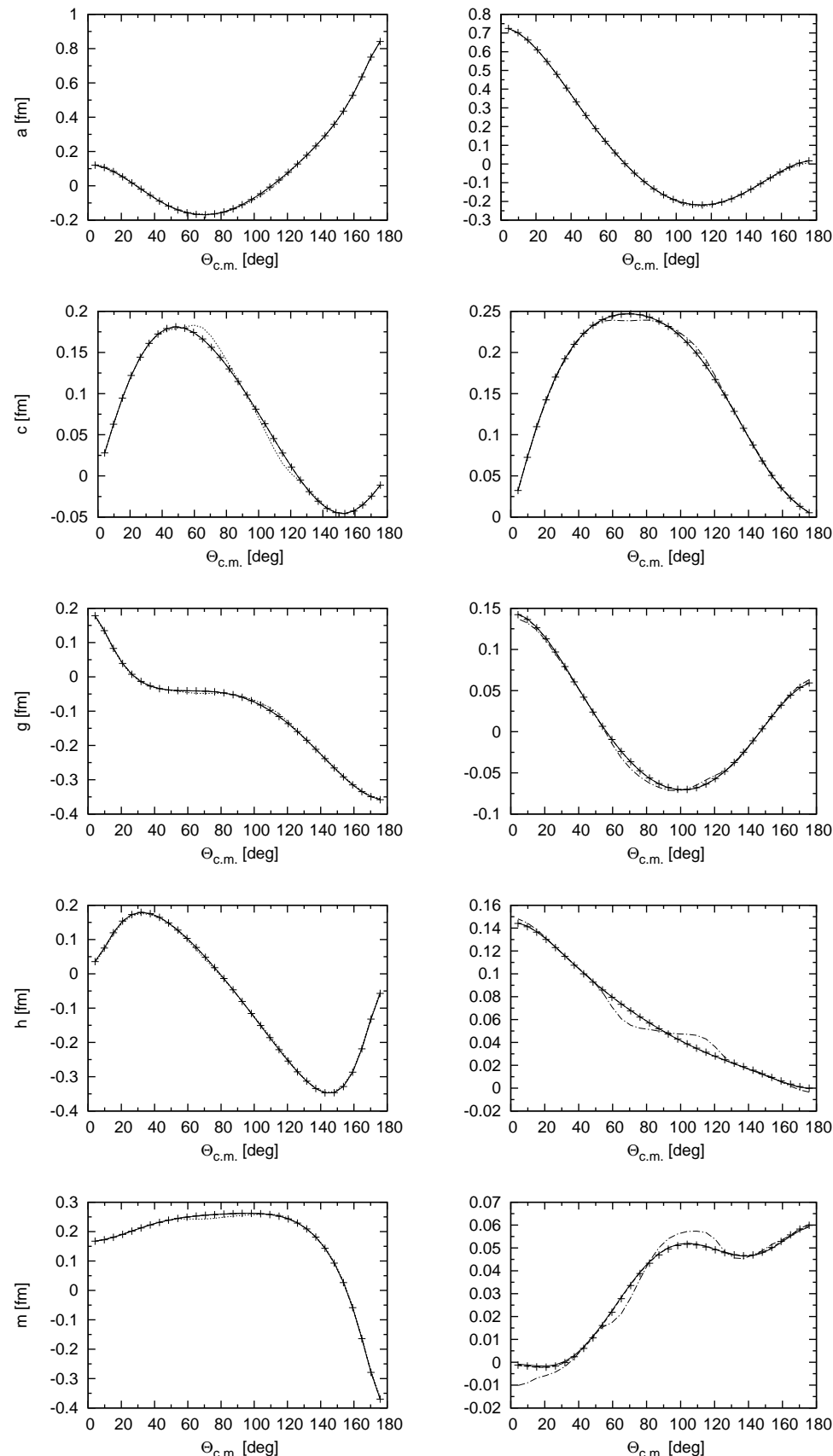

Fig. 3 The Wolfenstein parameters for neutron-proton scattering for projectile laboratory kinetic energy $300 \mathrm{MeV}$ calculated with the chiral NNLO potential [19. The left panels show the real parts of the amplitudes, whereas the imaginary parts are displayed in the right panels. Crosses and lines show results of the same types of calculations as in Fig. 1. 

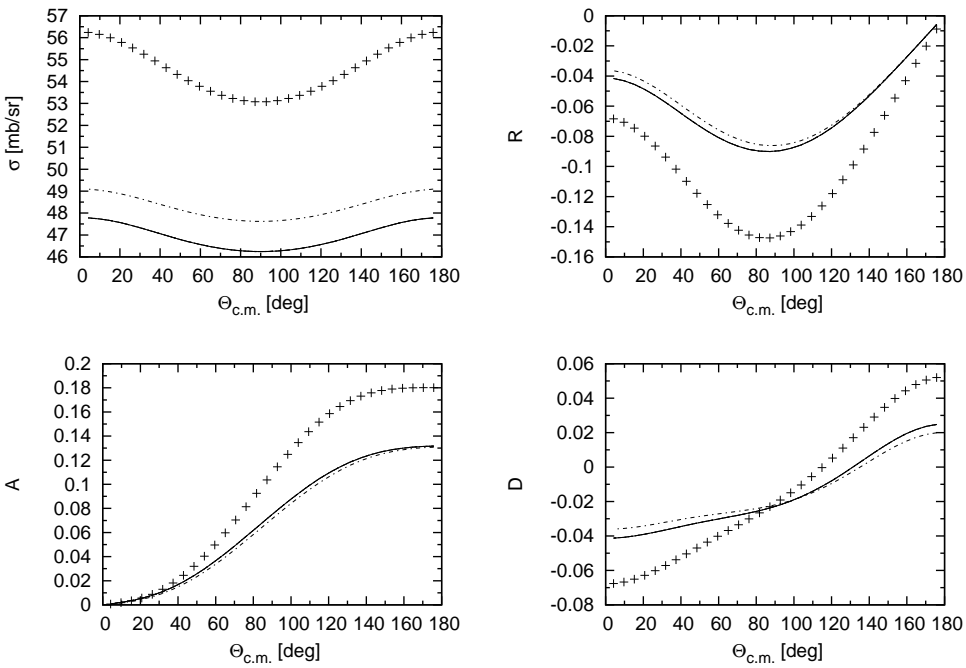

Fig. 4 The convergence of results obtained with the iterative method using different numbers of iterations for neutron-neutron (left panel) and neutron-proton (right panel) scattering observables for the projectile laboratory kinetic energy of $13 \mathrm{MeV}$ and the chiral NNLO potential [19. Crosses show the predictions with 6 iterations. Dash-dotted, double-dotted, dotted, dashed and solid lines display the results calculated with $8,10,12,14$ and 20 iterations, respectively. The last three lines overlap.
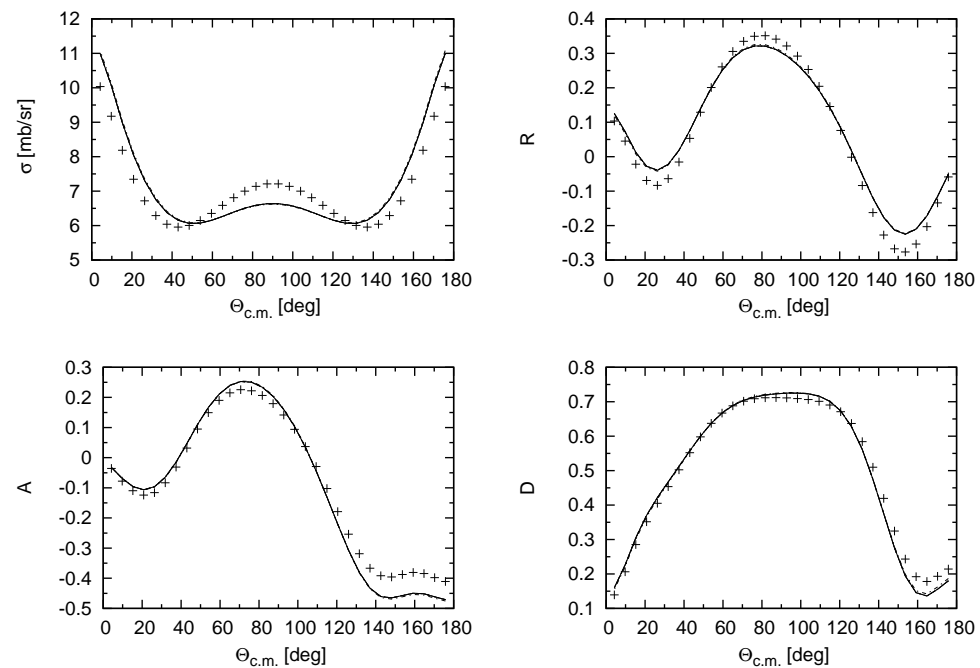

Fig. 5 The same as in Fig. 4 for the projectile laboratory kinetic energy of $300 \mathrm{MeV}$. 

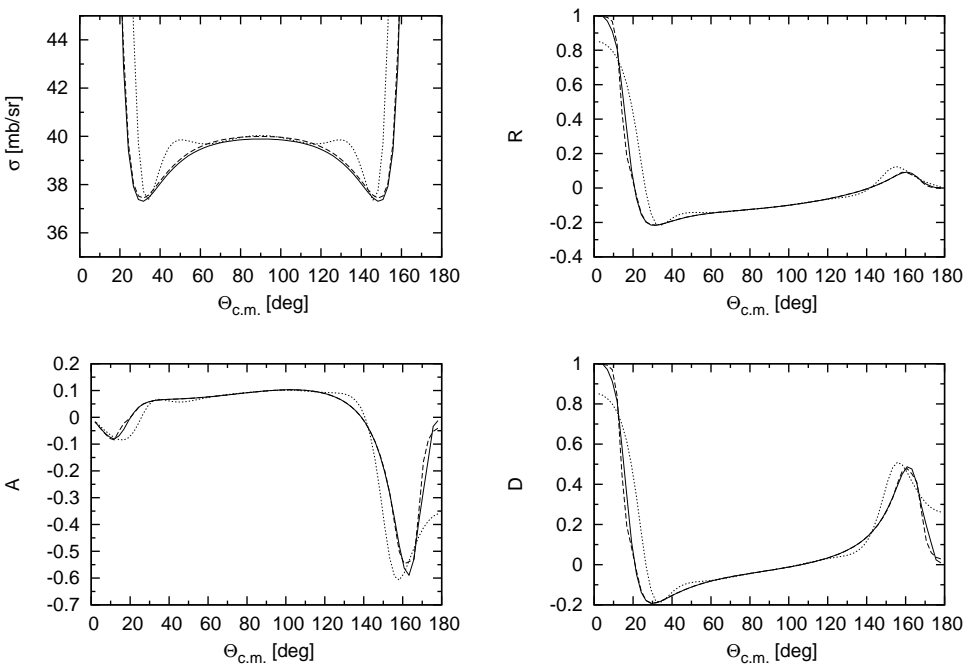

Fig. 6 Selected observables for the proton-proton system (including the Coulomb force) at the projectile laboratory kinetic energy $13 \mathrm{MeV}$ as a function of the centerof-mass angle $\theta_{c . m}$. for the chiral proton-proton NNLO potential 19,25. Results are obtained by solving the LS equation using the matrix inversion method and applying the exponential screening, $s_{1}(r ; n, R)$, to the Coulomb. Dotted, dashed and solid lines show the results with the screening parameter $R=20,60$ and 120 $\mathrm{fm}$, respectively, and $n=4$.
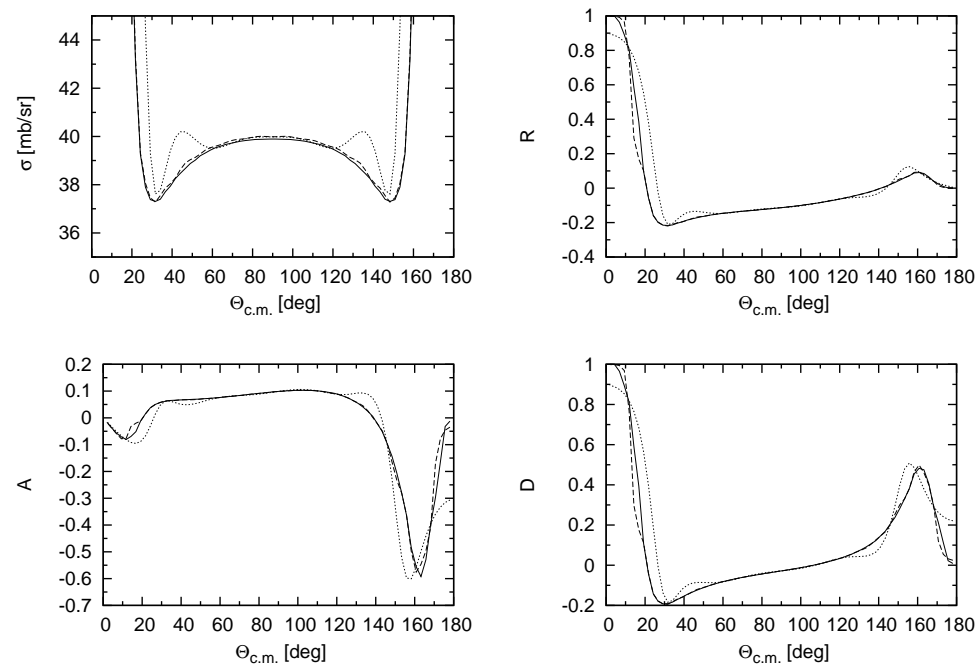

Fig. 7 The same as in Fig. 6 with the localized (sine function) screening (29), applied to the Coulomb force. Dotted, dashed and solid lines show the results with the screening parameter $R=10,30$ and $55 \mathrm{fm}$, respectively. 

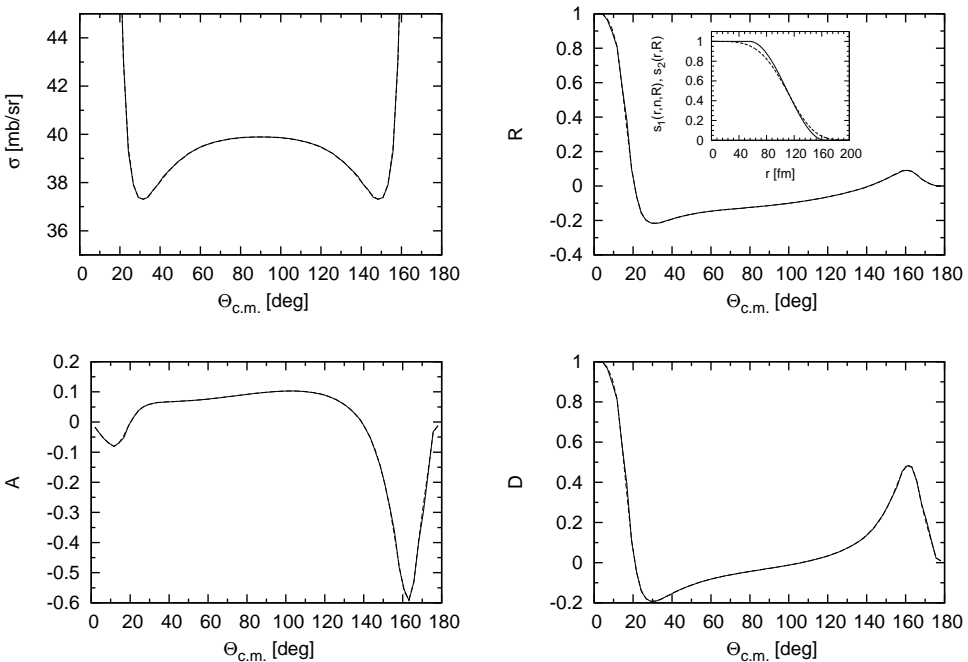

Fig. 8 Selected observables for the proton-proton system (including the Coulomb force) at the projectile laboratory kinetic energy $13 \mathrm{MeV}$ as a function of the centerof-mass angle $\theta_{c . m}$. for the chiral proton-proton NNLO potential 19,25. Results with the $s_{1}(r ; n=4, R=120 \mathrm{fm})$ screening (dashed lines) are compared with the predictions based on the $s_{2}(r ; R=55 \mathrm{fm})$ screening (solid lines). In the inset the two screening functions are compared: the dashed (solid) line shows the $s_{1}\left(s_{2}\right)$ function.
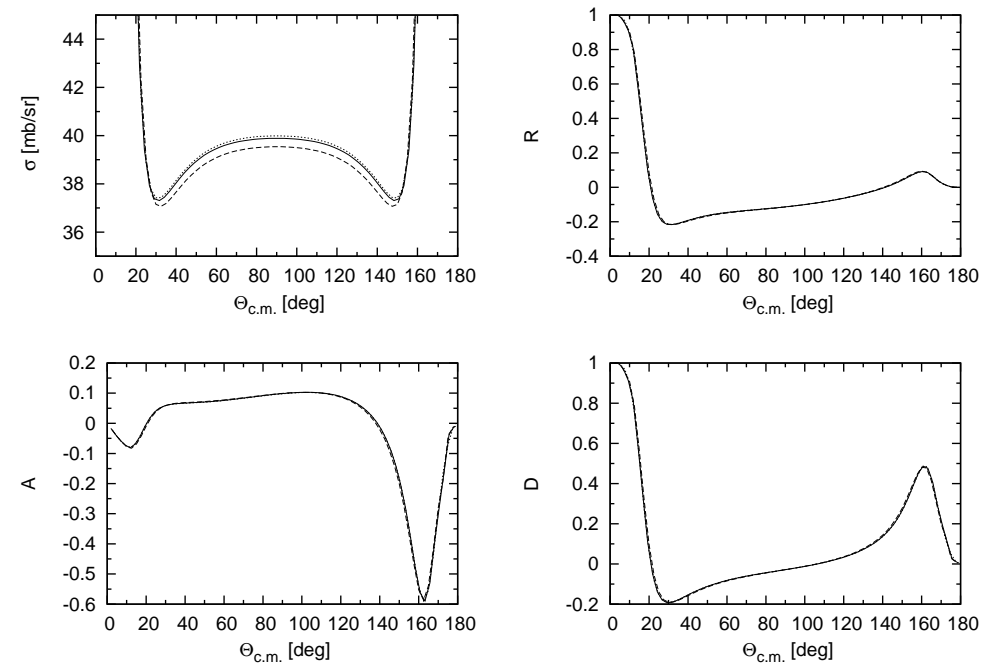

Fig. 9 Selected observables for the proton-proton system (including the Coulomb force) at the projectile laboratory kinetic energy $13 \mathrm{MeV}$ as a function of the centerof-mass angle $\theta_{c . m}$. for the chiral proton-proton NNLO potential [19,25]. Fully 3D results with the $s_{1}(r ; n=4, R=120 \mathrm{fm})$ screening (solid lines) are compared with the predictions based on two methods used in Ref. 27]: the method combining a 3D Coulomb matrix (for the same screening $s_{1}$ ) with PWD calculations (dashed lines) and the Vincent-Phatak method 29] (dotted lines). 

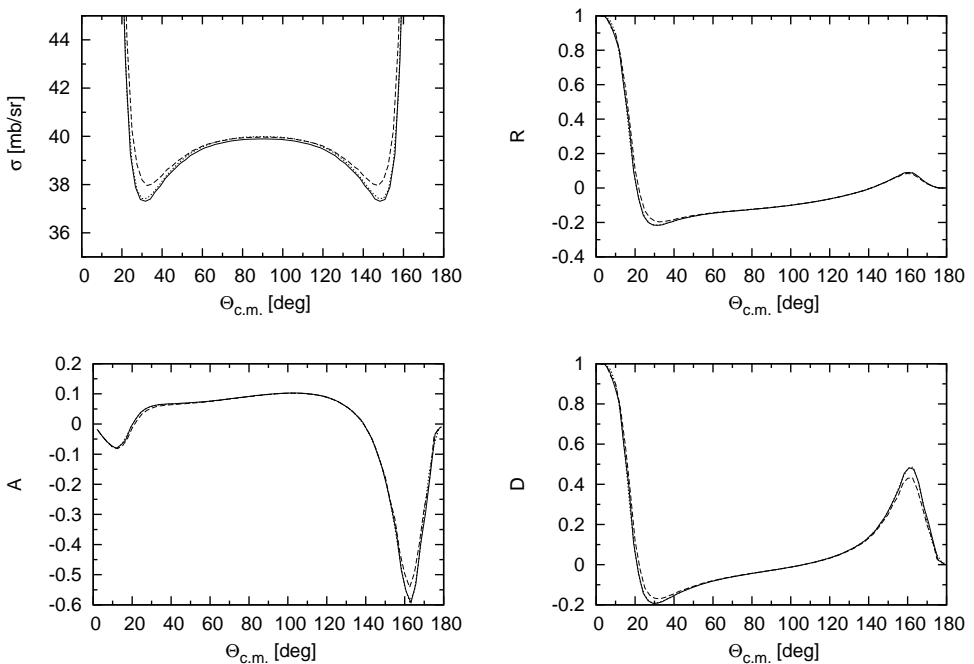

Fig. 10 The same as in Fig. 9 but the $s_{2}(r ; R=55 \mathrm{fm})$ screening 8 ] is used in the $3 \mathrm{D}$ calculations involving the Coulomb force. 